\title{
O papel da gestão de enfermagem na implementação da meta de cirurgia segura: uma
}

\section{revisão de literatura}

\author{
The role of nursing management in implementing the safe surgery goal: a literature review \\ El papel de la gerencia de enfermería en la implementación del objetivo de cirugía segura: una \\ revisión de la literatura
}

Recebido: 02/10/2021 | Revisado: 09/11/2021 | Aceito: 10/11/2021 | Publicado: 14/11/2021

\author{
Dmyttri Kussov Lobato Azevedo \\ ORCID: https://orcid.org/0000-0003-2480-0849 \\ Centro Universitário da Amazônia, Brasil \\ E-mail: faleirodmy@gmail.com \\ Crizoleide Melo Paranatinga da Silva \\ ORCID: https://orcid.org/0000-0001-7884-3671 \\ Centro Universitário da Amazônia, Brasil \\ E-mail: melocrizoleide@gmail.com \\ Adria Leitão Maia \\ ORCID: https://orcid.org/0000-0002-4173-1374 \\ Centro Universitário da Amazônia, Brasil \\ E-mail: adria-maia@hotmail.com
}

\begin{abstract}
Resumo
O indivíduo é exposto à intervenções cirúrgicas está sujeito a diversos tipos de riscos e complicações relacionados ao procedimento, que podem favorecer o aumento da mortalidade. Objetiva - se discorrer a respeito da implementação do protocolo de cirurgia segura, destacando o papel do enfermeiro para qualidade e eficácia do processo. Trata - se de uma revisão de literatura, de caráter descritivo; utilizando artigos de periódicos científicos da área da saúde, teses e dissertações, disponíveis em: SCIELO, LILACS, MEDLINE; nos idiomas português, inglês e espanhol. Utilizando o método de Bardin para análise de conteúdo. O Checklist do paciente seguro é recurso simples, de fácil aplicação e baixo custo, o mesmo identifica, compara, facilita a comunicação da equipe cirúrgica e analisa itens e procedimento do transoperatório, de modo a reduzir falhas no processo; esse protocolo está associado a melhores resultados no pósoperatório, com redução da mortalidade e de complicações cirúrgicas. Por ser a enfermagem uma profissão da área da saúde, se torna necessário que a enfermagem realize suas práticas baseadas em evidências científicas. Avalia-se que a educação permanente pode ser uma estratégia que vem favorecer o processo de capacitação, em conjunto com interesse e à responsabilidade profissional para a realização de uma assistência mais segura.
\end{abstract}

Palavras-chave: Segurança do paciente; Check List de segurança do paciente; Centro cirúrgico.

\begin{abstract}
The individual is exposed to surgical interventions and is subject to different types of risks and complications related to the procedure, which can favor an increase in mortality. Objective - to discuss the implementation of the safe surgery protocol, highlighting the role of the nurse for the quality and effectiveness of the process. It is a descriptive literature review; using articles from scientific journals in the health field, theses and dissertations, available in: SCIELO, LILACS, MEDLINE; in Portuguese, English and Spanish. Using the Bardin method for content analysis. The Safe Patient Checklist is a simple resource, easy to apply and low cost, it identifies, compares, facilitates the communication of the surgical team and analyzes items and intraoperative procedures, in order to reduce process failures; this protocol is associated with better postoperative results, with a reduction in mortality and surgical complications. As nursing is a profession in the health area, it is necessary for nursing to carry out its practices based on scientific evidence. It is estimated that continuing education can be a strategy that favors the training process, together with interest and professional responsibility to provide safer care.
\end{abstract}

Keywords: Patient safety; Patient safety checklist; Surgery center.

\section{Resumen}

El individuo está expuesto a intervenciones quirúrgicas y está sujeto a diferentes tipos de riesgos y complicaciones relacionados con el procedimiento, que pueden favorecer un aumento de la mortalidad. Objetivo - discutir la implementación del protocolo de cirugía segura, destacando el papel de la enfermera para la calidad y efectividad del proceso. Es una revisión descriptiva de la literatura; utilizando artículos de revistas científicas del área de la salud, tesis y disertaciones, disponibles en: SCIELO, LILACS, MEDLINE; en portugués, inglés y español. Utilizando el método Bardin para el análisis de contenido La Lista de Verificación del Paciente Seguro es un recurso simple, fácil de aplicar y 
de bajo costo, identifica, compara, facilita la comunicación del equipo quirúrgico y analiza ítems y procedimientos intraoperatorios, con el fin de reducir fallas en los procesos; este protocolo se asocia a mejores resultados postoperatorios, con reducción de la mortalidad y las complicaciones quirúrgicas. Como la enfermería es una profesión en el área de la salud, es necesario que la enfermería lleve a cabo sus prácticas con base en la evidencia científica. Se estima que la educación continua puede ser una estrategia que favorezca el proceso de formación, junto con el interés y la responsabilidad profesional por brindar una atención más segura.

Palabras clave: Seguridad del paciente; Lista de verificación de seguridad del paciente; Centro cirúrgico.

\section{Introdução}

O indivíduo é exposto à intervenções cirúrgicas está sujeito a diversos tipos de riscos e complicações relacionados ao procedimento, que podem favorecer o aumento da mortalidade. Das complicações que ocorrem intra e pós operatória, Sales et al. (2015) afirmam que desses eventos 50\% poderiam ser evitadas.

De acordo com a Organização Mundial de Saúde (OMS), muitas cirurgias são realizadas nos mais diferentes cenários, contudo, esses procedimentos precisam estar de acordo com os princípios de segurança e qualidade. Diante desta necessidade urgente, foi lançado o "Programa Cirurgias Seguras Salvam Vidas", da OMS, este recurso pretende contribuir para a redução de danos através da utilização da lista de verificação de cirurgia segura ou checklist, aplicado mundialmente (World Health Organization, 2009).

Com a iniciativa da OMS, e objetivando ofertar um cuidado de qualidade, o Ministério da Saúde Brasileiro estabeleceu o protocolo de cirurgia segura no ano de 2013, as recomendações são para a utilização da lista de verificação de segurança cirúrgica, este instrumento auxiliar na melhoria do cuidado aos pacientes que realizarão procedimentos cirúrgicos nos diversos hospitais no país (Brasil, 2013).

O Checklist do paciente seguro é recurso simples, de fácil aplicação e baixo custo, o mesmo identifica, compara, facilita a comunicação da equipe cirúrgica e analisa itens e procedimento do transoperatório, de modo a reduzir falhas no processo (Brasil, 2013). De acordo com Sales et al. (2015), esse protocolo está associado a melhores resultados no pós-operatório, com redução da mortalidade e de complicações cirúrgicas.

Dessa forma se faz necessário conhecer mais a respeito do tema afim de proporcionar aos familiares, pacientes e usuários do sistema de saúde, um ambiente seguro; assim como criar a cultura de segurança dentro das instituições de saúde (Gutierres et al., 2018).

O presente estudo tem por objetivo discorrer a respeito da implementação do protocolo de cirurgia segura, destacando o papel do enfermeiro para qualidade e eficácia do processo.

\section{Metodologia}

Este é um estudo de caráter descritivo, tratando - se de uma revisão de literatura, onde foram utilizadas teses e dissertações, artigos de periódicos científicos da grande área das ciências da saúde, manuais e protocolos de instituições de saúde disponíveis nas seguintes bases de dados científicos: SCIELO (Scientific Eletronic Library Online), LILACS (Literatura Latino Americana e do Caribe em Ciências da Saúde), BVS (Biblioteca virtual da Saúde), MEDLINE (Medical Literature Analysis and Retrieval System Online).

Os idiomas usados como filtros de seleção dos materiais para construção desta obra são: português, inglês e espanhol; usando os seguintes descritores: Segurança do Paciente, Check List de Segurança do Paciente, Centro Cirúrgico.

Como critérios de inclusão foram estabelecidos os seguintes: estar escrito nos idiomas anteriormente definidos, apresentar relação com o tema, terem sido publicados entre os anos de 2008 e 2020. Quanto aos critérios de exclusão: foram excluídos os textos incompletos, literaturas sem referência de autoria, com versões apenas em resumos, assim como não atender aos critérios de inclusão previamente descritos. 
Para o processo de análise de dados obtidos, foi utilizado o Método de Bardin; tal obra é destaque para análise de conteúdo. Segundo Bardin (2012), a análise de conteúdo é um conjunto de técnicas de análise de comunicações, que se utiliza de procedimentos sistemáticos e objetivos de descrição do conteúdo das mensagens. Ou seja, constituído por fases ou etapas que tem como objetivo sintetizar os achados. Silva (2021) ressalta a importância da utilização da técnica de análise de conteúdo para a construção de revisões de literaturas:

\begin{abstract}
"Expressam a atitude de desvendar aspectos comunicacionais não evidentes, de ultrapassar as barreiras da compreensão superficial, de transpor os limites expressos das mensagens para revelar novas dimensões, possibilidades de interpretação e, até mesmo, descobrir fatos ou circunstâncias que remontam às origens e que deram causa aos discursos. Para Bardin, os objetivos da análise de conteúdo são, de maneira geral, dois: ultrapassagem da incerteza e enriquecimento da leitura. O primeiro representa o desejo de rigor do método, de forma que seus resultados possam ser aceitos e verificados pela comunidade científica. Por sua vez, o segundo é relacionado ao ímpeto de descobrir, desvendar, aprofundar, ultrapassar o expressamente contido no evento comunicativo" (Silva, 2021, p.43-44)
\end{abstract}

As etapas de análises propostas pela autora são respectivamente: pré - análise, exploração sistemática dos documentos obtidos e tratamento dos resultados. As fases de análise dos artigos obtidos para a construção desta obra, estão descritos na Tabela 1.

Tabela 1. Síntese do método de análise de conteúdo de Bardin.

\begin{tabular}{c|l}
\hline \multicolumn{2}{c}{ MÉTODO DE ANÁLISE DE CONTEÚDO } \\
\hline $\begin{array}{c}\text { FASE I } \\
\text { Pré - análise }\end{array}$ & $\begin{array}{l}\text { Seleção dos materiais que tem relação com o objeto de estudo, embasamento argumentativos } \\
\text { para os resultados obtidos. Nesta fase são aplicados os critérios de inclusão e exclusão, para } \\
\text { facilitar a obtenção de dados que serão realmente aproveitados. }\end{array}$ \\
\hline $\begin{array}{c}\text { FASE II } \\
\text { Exploração do } \\
\text { material }\end{array}$ & $\begin{array}{l}\text { Estudo e leitura minuciosa dos materiais obtidos. Nesta fase são avaliação os escritos e } \\
\text { selecionados os que serão necessários para sustentar do tema de estudo, assim como os que } \\
\text { serão utilizados para fazer correlação com a proposta do tema em questão. }\end{array}$ \\
\hline $\begin{array}{c}\text { FASE III } \\
\text { Tratamento dos } \\
\text { resultados }\end{array}$ & $\begin{array}{l}\text { Validação dos dados analisados. Relação dos objetivos com os resultados, e possível } \\
\text { surgimento de novas hipóteses. }\end{array}$ \\
\hline
\end{tabular}

Fonte: Azevedo, Silva e Maia (2021).

\title{
3. Resultados e Discussão
}

A coleta de dados se deu por meio de pesquisas via Internet, em sites de periódicos e repositórios virtuais de instituições educacionais, conforme descrito na metodologia. Desse modo, inicialmente foram selecionados 32 artigos usando os seguintes descritores acima mencionados, dos quais apenas 15 estavam de acordo com os critérios de inclusão.

\subsection{Eventos adversos evitáveis relacionados a cirurgias}

Para melhor entender a temática em questão se faz necessário compreender o seguinte conceito de eventos adversos: “[...] A Organização Mundial da Saúde (OMS) classifica eventos adversos como incidentes que resultam em danos não intencionais decorrentes da assistência e não relacionados à evolução natural da doença de base do paciente [...]” (Fiocruz, 2012).

Monitorar as complicações operatórias tem sido um desafio no Brasil, de acordo com Sousa et al. (2020), não utilização ou a utilização incorreta de sistemas padrão de classificação e caracterização de complicações, se constitui com a mais importante limitação no que concerne a notificação de eventos adversos relacionados a procedimentos cirúrgicos.

Em sua revisão integrativa o mesmo autor ainda observou que os estudos que exploraram as complicações limitaram-se a descrever arbitrariamente as complicações como "grave", "maior" ou "menor", o que dificulta comparar resultados na literatura de modo geral, este mesmo acontecimento também foi confirmado por Tevis e Kennedy (2013), em um estudo anterior. 
Em seu estudo, Sousa et al. (2020), observou as complicações infecciosas mais foram evidenciadas: infecção relacionada ao sitio cirúrgico/ferida operatória, hemorragia pós-operatória, sendo que em estudo realizado com pacientes submetidos à nefrolitotomia percutânea, esse valor chegou a 12,5\% dos pacientes. O acontecimento dessas complicações tem sido fortemente associado a necessidade de readmissões e/ou mortalidade elevada.

Mendes et al. (2013), destacou que relacionados a procedimentos cirúrgicos e/ou anestésicos representaram $20 \%$ dos eventos adversos que poderiam ser evitados, ficando atrás apenas de Infecções associadas aos cuidados da saúde (24\%); tais eventos adversos com relação a procedimentos cirúrgicos adicionaram mais 79 dias às internações, para resolver as complicações consequentes.

Diante disso se pode inferir os prejuízos trazidos ao sistema de saúde, sem deixar de mencionar, os impactos gerados ao próprio paciente e aos seus familiares, que por vezes tem que lidar com o luto pela perda de seu ente querido.

No que diz respeito ao local onde mais correm eventos adversos, o centro cirúrgico também se destacou, ficando em segundo lugar entre os maiores índices de eventos evitáveis (31\%). Em relação a falhas da prevenção da ocorrência, 72 \% dos eventos adversos ocorreram porque os profissionais envolvidos, não tomaram as precauções adequadas.

Diante do exposto, podemos mensurar a importância de ser ter um instrumento que seja capaz de mitigar esses acontecimentos, e que seja sistemático, ou seja, que seja realmente realizado fase por fase, e posteriormente avaliado afim de encontrar pontos frágeis, em seguida fazer implementações no processo de melhoria da assistência prestada.

\subsection{O protocolo}

Segundo Jordão et al. (2019), o checklist ou protocolo da Cirurgia Segura foi elaborado por peritos internacionais reunidos pela Organização Mundial da Saúde, e adaptado a realidade de diversos países, dentre eles o Brasil. O checklist de cirurgia segura da OMS tem três momentos, conforme descrito na Figura 1: Entrada/Identificação (antes da incisão cirúrgica), Time Out/confirmação ou pausa (antes da incisão -_pausa com a presença de todos os membros da equipe na sala cirúrgica) e saída/registro (antes de o paciente deixar o centro cirúrgico) (Silva et al., 2020).

De acordo com os elementos do protocolo Cirurgia Segura podem ser adequados de acordo com às necessidades de cada instituição de saúde, alguns elementos que merecem atenção estão dispostos no Quadro 1. Uma recomendação importante estabelecida pela OMS é que o checklist não seja muito extenso, tornando maçante a sua realização, além disso, também é recomendado que perguntas não sejam eliminadas (Opas, 2009). 
Figura 1. Lista de verificação de segurança cirúrgica (primeira edição).

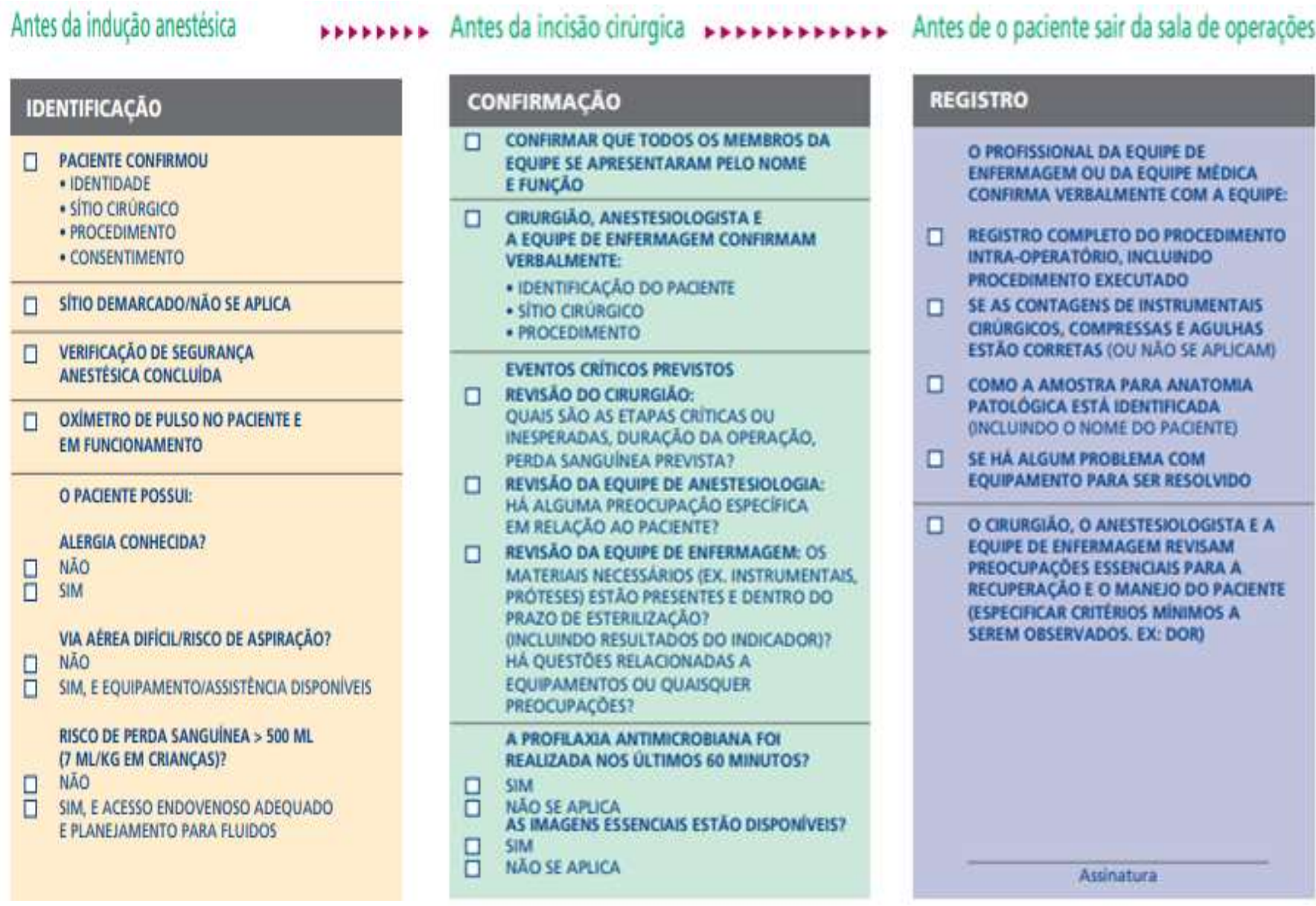

Fonte: Opas (2009).

Em seu estudo, Fonseca (2010), identificou redução do número de mortes durante o procedimento cirúrgico diminuiu de $1,5 \%$ para $0,8 \%$ e as complicações pós-cirurgia caíram de $11 \%$ para $7 \%$. O mesmo autor ainda observou queda nas taxas de infecção e no retorno não planejado ao centro cirúrgico, o que se confirma na obra de Neri (2016).

Quadro 1. Cirurgia segura.

10 PONTOS DE ATENÇÃO PARA A CIRURGIA SEGURA

1 - Certificar - se de que é o paciente certo e sítio cirúrgico correto

2 - Proteger o paciente da dor, minimizando os riscos inerentes do procedimento anestésico;

3 - Ter capacidade para reconhecer dificuldades respiratórias e um plano de ação pronto

4 - Preparar -se para identificar e agir em casos de grande perda sanguínea

5 - Evitar induzir reações alérgicas ou à medicação que tragam riscos ao paciente

6 - Usar métodos para minimizar o risco de infecções de sítio cirúrgico

7. Evitar a retenção de compressas ou instrumentos em feridas cirúrgicas

8 - Identificar de maneira precisa todos os espécimes cirúrgicos

9 - Comunicar e trocar informações críticas sobre o paciente

10. Cabe a hospitais e sistemas públicos de saúde estabelecer vigilância de rotina de resultados, volumes e capacidade cirúrgica

Fonte: Azevedo, Silva e Maia (2021). 


\subsection{O enfermeiro gestor na implementação da cirurgia segura}

De acordo com Maurício et al. (2019), o enfermeiro como gestor da equipe de enfermagem, tem um papel fundamental tanto na realização dos cuidados de sua competência, quanto na supervisão daqueles que são prestados pelos técnicos e auxiliares de enfermagem em um bloco cirúrgico, bem como na participação da equipe multiprofissional.

Sales et al. (2015), ressalta o papel do enfermeiro na gestão do protocolo de cirurgia segura, pois geralmente o manuseio do checklist é de sua responsabilidade. Os mesmos autores, ainda corroboram que o uso desse instrumento de registro pelo enfermeiro tem o potencial de reduzir drasticamente a ocorrência de efeitos adversos, facilitando o seu trabalho e diminuindo os custos hospitalares.

No que se refere ao paciente, o uso do checklist se constitui em uma garantia de que o mesmo passará por um procedimento cirúrgico seguro e poderá retomar suas atividades normais e qualidade de vida preservada, no que diz respeito eventos adversos (Lopes et al., 2018).

Logo, a falta de conhecimento por parte dos enfermeiros dificulta a seu desempenho no que tange ao julgamento clínico das respostas do paciente. Em contra ponto, quanto maior a afinidade do enfermeiro, no reconhecimento do processo e da importância do checklist, e das intervenções para mitigar falhas no processo, maior será a sua habilidade, acurácia e agilidade em intervir de maneira assertiva (Pazetti, 2020).

Um dos problemas encontrados para a realização do Checklist de forma eficaz é a adesão dos profissionais envolvidos com a sua aplicação, ou seja, os profissionais de enfermagem e a equipe anestésico - cirúrgica; para Pazetti et al. (2020) a resistência e dificuldades no preenchimento seja devido à falta de capacitação e sensibilização quanto a política de segurança do paciente cirúrgico para toda a equipe cirúrgica e da grande rotatividade da equipe de profissionais.

\section{Considerações Finais}

O preparo da equipe de enfermagem e o engajamento da equipe anestésico- cirúrgico aliado ao reconhecimento dos processos que envolvem a realização do checklist do paciente seguro, bem como a capacitação necessária para sua aplicação, evidenciam o zelo por uma assistência de qualidade, o que repercute positivamente tanto para a instituição quanto para usuário do serviço de saúde.

Avalia-se que a educação permanente pode ser uma estratégia que vem favorecer o processo de capacitação, em conjunto com interesse e à responsabilidade profissional para a realização de uma assistência mais segura. Dessa forma, exige-se do profissional que o mesmo tenha postura ativa, participativa e transformadora, o que é essencial para um gestor; uma vez que um serviço de qualidade é reafirmado quando é realizado com sensibilidade, dedicação e ciência.

Já é sabido que o protocolo do Paciente Seguro é uma estratégia com resultados garantidos, uma vez que seja executado passo a passo da maneira correta, o que faz do papel do enfermeiro um elo para a corrente da segurança do paciente. Não houve a pretensão esgotar os estudos voltados para o tema, descrevendo uma visão geral do papel do enfermeiro gestor na implementação da meta de cirurgia segura. Tendo como maior propósito a contribuição para os estudos acerca do tema proposto, como referência nas pesquisas em saúde e para o incentivo à mais estudos sobre o tema.

A preocupação das instituições de saúde com a segurança do paciente denota a qualidade da assistência prestada, suge se que este tema seja mais explorado através da aplicação e adequação desse protocolo nas diferentes regiões do país, afim de se obter um panorama a respeito da prática do dia a dia com este instrumento e a experiência de outras realidades. Outra vertente seria avaliar o conhecimento da equipe cirúrgica sobre o passo a passo do protocolo, afim de se obter um feedback sobre as fragilidades e os pontos fortes para a aplicação do protocolo diariamente. 


\section{Agradecimentos}

Agradecemos primeiramente a Deus pela saúde e coragem que nos tem dado durante esses anos de graduação, agradecemos também a nossa orientadora Adria, pois sem ele nada disso seria possível.

\section{Referências}

Bardin, L. (2012). Análise de conteúdo: a revisão de Laurence Bardin. Revista Eletrônica de Educação. Programa de Pós-graduação em Educação. 6(1). http://dx.doi.org/10.14244/\%2519827199291.

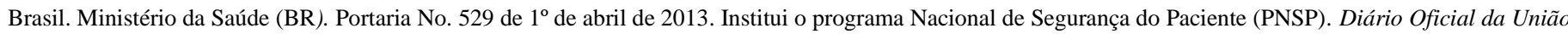
http://www20.anvisa.gov.br/ segurancadopaciente/ index.php/publicações.

Fiocruz. (2012). Aprimorando as práticas de saúde. Proqualis: eventos adversos. https://portal.fiocr uz.br/noticia/proqualis-ganha-pagina sobre-eventos-adversos.

Fonseca, S. N. S. (2010). Cirurgia Segura. 15ª Jornada de Controle de Infecção Hospitalar. A Relevância do Enfermeiro no Protocolo de Cirurgia Segura Salva Vidas. Revisão da Literatura. http://www.cve.saude.sp.gov.br/htm/ih/pdf/ih10_hsfjornada.pdf.

Gomes, L. C., Dutra, K. E., \& Pereira, A. L. S. (2014). O Enfermeiro no Gerenciamento do Centro Cirúrgico. Revista Eletrônica da Faculdade Metodotista Granbery; 16:01-21. http://re.granbery.edu.br/artigos/NTEy.pdf.

Gutierres, L. S., Santos, J. L. G., Peiter, C. C., Menegon, F. H. A., Sebold, L. F., \& Erdmann, A. L. (2018). Good practices for patient safety in the operating room: nurses' recommendations. Revista Brasileira de Enfermagem. 71(6):2775-82. http://dx.doi.org/10.1590/0034-7167-2018-0449.

Jordão, K. M. D.; Soares, R. A. Q.; Fernandes, I. T. G. P.; Nascimento, A. L.; Ferreira, M. Z. J.; \& Santos, S. M. (2019). Atuação do enfermeiro nos protocolos de cirurgia segura. Revista de Saúde Coletiva. 9(49). http://www.revistas.mpmcomunicacao.com.br/index.php/saudecoletiva/article/view/122/106.

Lopes, M. C. R., Silva, L. F., Barros, T. S., Martins, F. J. G., \& Farias, M. S. (2018). Atuação da enfermagem no processo de cirurgia segura. Revista Tendências da Enfermagem Profissional. Revista Tendências da Enfermagem Profissional. http://www.coren-ce.org.br/wp-content/uploads/2020/01/Atua\%C3\%A7\%C3\%A3 o-da-enfermagem-no-processo-de-cirurgia-segura.pdf.

Maurício, Í.H., Souza, L. K. S., Santos, T. T., Andrade, V. E. M., \& Godinho, E. M A. (2019). Cirurgia Segura: um instrumento de enfermagem voltado para a segurança do paciente cirúrgico. Univale. https://www.univale.br/wp-content/uploads/2019/12/ENFER.-2019_2-CIRURGIA-SEGURA-UM-INSTRUMENTODE-ENFERMAGEM...-\%C3\%8DCCARO.LORENA.-THASSYLA.-VIN\%C3\%8DCIUS.pdf .

Mendes, W., Pavão, A.L., Martins, M., Moura, M. L., \& Travassos, C. (2013). The feature of preventable adverse events in hospitals in the State of Rio de Janeiro, Brazil. Revista Associação Médica Brasileira (1992). 59(5):421-8. 10.1016/j.ramb.2013.03.002.

Mendes, W., Martins, M ., Rozenfeld, S., \& Travassos, C. The assessment of adverse events in Brazilian hospitals. International Journal Quality Health Care. 2009. $10.1093 /$ intqhe / mzp022.

Neri, M. F. A. (2016). Cirurgia Segura: Atuação do Enfermeiro em Bloco Cirúrgico. Programa de Pós Graduação em Enfermagem da Universidade de Pernambuco. Faculdade Integrada De Pernambuco - Facipe Bacharelado Em Enfermagem. Recife - Brasil. https://openrit.grupotiradentes.com/xm lui/bitstream/handle/set/1802/TCC_Merari\%20Ferreira\%20A\%20Neri.pdf?sequence=1.

Organização Mundial da Saúde -OMS. (2008). Safe surgery saves lives: second global patient safety challenge. Publications of the World Health Organization can be obtained from WHO Press, World Health Organization.: https://www.who.int/patient safety/safesurgery/knowledge_bas e/SSS L_Brochure_finalJun08.pdf.

Organização Pan-Americana da Saúde - OPAS. (2009). Segundo desafio global para a segurança do paciente. Ministério da Saúde, Agência Nacional de Vigilância Sanitária. http://bvsms.saude.gov.br/bvs/publicacoes/seguranca_paciente_cirurgias_seguras_guia.pdf .

Panzetti., J. M. L. S., Vasconcelos, L. A., Araújo, M. A. G., Oliveira, V.M. L. P., Castilho, F. N. F., Oliveira, J. S., Costa, T. M., Rodrigues, R. P., Ramos, A. M. P. C., \& Maia, G. C. (2020). Adesão da equipe de enfermagem ao protocolo de cirurgia segura. Revista Eletrônica Acervo Saúde. 12(2) https://doi.org/10.252 48/reas.e2519.2020. Página 1d.

Sales, F. S., Neres, R. G., \& Azevedo, E. R. (2015). A relevância do enfermeiro no protocolo de cirurgia segura salva vidas: revisão da literatura. Faculdades Promove de Brasília. http://nippromove.hospedagemdesites.ws/anais_simposio/arquivos_up/documentos/artigos/bacfddcb4465c1ef59e9463e2b63c334.pdf.

Silva, A. M. R., Silva, I. T. C., Rocha, G S., \& Teixeira, E. (2020). Protocolo de cirurgia segura: análise da produção e execução em dois hospitais terciários. Revista SOBECC, 25(3). https://doi.org/10.5327/Z14144425202000030002.

Silva, N. B. F. (2021). As Interfaces da Diversidade, do Patrimônio e da Globalização a partir das Produções Científicas. Campo Grande, Mato Grosso do Sul: Life Editora, 2021. 43-44.

Sousa, A. F. L., Bim, L. L., Hermann, P. R. S., Fronteira, I., \& Andrade, D. (2020). Complicações no pós-operatório tardio em pacientes cirúrgicos: revisão integrativa. Revista Brasileira de Enfermagem. 73(5):e20190290. http://dx.doi.org/10.1590 /0034-7167-2019-0290. 
Research, Society and Development, v. 10, n. 14, e584101422711, 2021

(CC BY 4.0) | ISSN 2525-3409 | DOI: http://dx.doi.org/10.33448/rsd-v10i14.22711

Tevis, S. E., \& Kennedy, G. D. (2013) Postoperative complications and implications on patient centered outcomes. Journal of Surgical Research. 181(1):106-13. 10.1016/j.jss.2013.01.032.

World Health Organization. (2009). WHO guidelines for safe surgery. Geneva: WHO. http://www.tngda.org/media/pdf/2960555885_safe\%20surgery.pdf. 